\title{
Clinicopathological and molecular characteristics of patients with hypermutant lung cancer: A retrospective cohort study
}

\author{
HONGBIN ZHANG $^{1}$, YUAN WANG ${ }^{1}$, QIAOXIA JI ${ }^{1}$, HONGMEI CAI ${ }^{1}$, XIANGCUN LIANG ${ }^{1}$, \\ JIONG XIE ${ }^{1}$, HUA LI $^{1}$, JUN WANG ${ }^{2}$, GUIYUN ZHU ${ }^{3}$, ERPENG TIAN ${ }^{4}$, LINGLING ZHU ${ }^{1}$, \\ MINGMING YUAN ${ }^{5}$, RONGRONG $\mathrm{CHEN}^{5}$ and MIN ZHAO ${ }^{1}$ \\ ${ }^{1}$ Department of Oncology, Hebei Chest Hospital, Research Center of Hebei Lung Cancer Prevention and Treatment, \\ Shijiazhuang, Hebei 050041; ${ }^{2}$ Department of Radiation Oncology, Fourth Hospital of Hebei Medical University, \\ Shijiazhuang, Hebei 050011; ${ }^{3}$ Department of Pathology; ${ }^{4}$ Molecular Biology Laboratory, Hebei Chest Hospital, \\ Shijiazhuang, Hebei 050041; ${ }^{5}$ Geneplus-Beijing, Beijing 102206, P.R. China
}

Received May 26, 2020; Accepted January 28, 2021

DOI: 10.3892/ol.2021.12590

\begin{abstract}
Tumor mutation burden (TMB) is an independent indicator used to select patients sensitive to immunotherapy. The present study aimed to investigate the clinicopathological and molecular characteristics of patients with hypermutant lung cancer to identify an economical, simple and complementary method for predicting TMB and immunotherapy responses. In total, 1,000 patients with lung cancer were randomly selected, and their samples were submitted to next-generation sequencing, with their TMB status reviewed. The threshold of hypermutation was set to 17.24 mutations (muts)/Mb. The proportion of smokers was higher in the hypermutant cohort $(n=67)$ compared with in the non-hypermutant cohort ( $\mathrm{n}=933 ; 85.1$ vs. $46.6 \%$; $\mathrm{P}<0.0001)$. Compared with in the non-hypermutant cohort, the proportion of squamous cell carcinoma cases and small cell lung cancer cases was higher in the hypermutant cohort (22.4 vs. $13.1 \%$ and 6.0 vs. $2.6 \%$, respectively). In addition, compared with in the non-hypermutant cohort, mutations in the low-density lipoprotein receptor-related protein $1 \mathrm{~B}$ were more frequently observed in the hypermutant cohort (67.2 vs. $14.3 \%$; $\mathrm{P}<0.0001)$. A similar trend was obtained for all genes tested, except for the EGFR
\end{abstract}

Correspondence to: Dr Min Zhao, Department of Oncology, Hebei Chest Hospital, Research Center of Hebei Lung Cancer Prevention and Treatment, 372 Shengli North Street, Shijiazhuang, Hebei 050041, P.R. China

E-mail: zhaomin20200526@163.com

Abbreviations: NSCLC, non-small cell lung cancer; SCLC, small cell lung cancer; ORR, objective response rate; TMB, tumor mutation burden; NGS, next-generation sequencing; MSI, microsatellite instability; DDR, DNA damage response; FFPE, formalin-fixed, paraffin-embedded; SNV, single nucleotide variant; InDel, insertions and deletions; DMG, differentially mutated gene

Key words: hypermutation, immunotherapy, lung cancer, next-generation sequencing, Notch signaling gene. Furthermore, in the hypermutant cohort, the prevalence of microsatellite instability was extremely high (9.0\%). The mutation frequency in DNA damage response (DDR) genes was notably higher in the hypermutant cohort, where several DDR-associated genes were enriched, compared with in the non-hypermutant cohort. The enrichment analysis revealed a strong association between mutations in Notch signaling and high TMB. To the best of our knowledge, the present study is the first to comprehensively investigate the clinical and genetic characteristics of patients with hypermutant lung cancer in a Chinese population. The results of the current study suggested that hypermutant lung cancer exerted distinctive clinical and genetic features, which may be used as complementary indicators for screening patients sensitive to immunotherapy.

\section{Introduction}

Lung cancer is the leading cause of cancer-associated death among males and females, accounting for an estimated 600,000 associated deaths in China in 2015 (1). Immune checkpoint blockade has emerged as a promising strategy in several malignancies, including both non-small cell lung cancer (NSCLC) and small cell lung cancer (SCLC) (2-6). The CheckMate-032 study investigated the efficacy of nivolumab, an anti-programmed cell death-1 (PD-1) antibody, alone or in combination with ipilimumab, an anti-cytotoxic T lymphocyte antigen-4 antibody in recurrent patients with SCLC, who progressed after one or more prior regimens (6). In the aforementioned study, the objective response rates (ORRs) were 10,23 and $19 \%$ for patients treated with $3 \mathrm{mg} / \mathrm{kg}$ nivolumab, $1 \mathrm{mg} / \mathrm{kg}$ nivolumab $+3 \mathrm{mg} / \mathrm{kg}$ ipilimumab and $3 \mathrm{mg} / \mathrm{kg}$ nivolumab $+1 \mathrm{mg} / \mathrm{kg}$ ipilimumab, respectively (6). Among the patients with metastatic NSCLC who progressed following platinum-based chemotherapy, nivolumab was associated with significantly longer median overall survival time compared with docetaxel [OS; non-squamous NSCLC, 12.2 vs. 9.4 months; hazard ratio (HR), $0.73 ; 95 \%$ CI, 0.59-0.89; $\mathrm{P}=0.002$; squamous NSCLC, 9.2 vs. 6.0 months; HR, 0.59; 95\% CI, 0.44-0.79; $\mathrm{P}<0.001]$ (7,8). Other immune checkpoint inhibitors, including pembrolizumab, an anti-PD-1 antibody 
[programmed death-ligand 1 (PD-L1)-positive NSCLC population, 10.4 vs. 8.5 months; HR, 0.71; 95\% CI, 0.58-0.88; $\mathrm{P}=0.0008]$, and atezolizumab, an anti-PD-L1 antibody (13.8 vs. 9.6 months; HR, 0.73; 95\% CI, 0.62-0.87; $\mathrm{P}=0.0003$ ), also exhibited improved OS compared with chemotherapy $(9,10)$. However, the response rate of immune checkpoint inhibitors was relatively low in unselected patients (5). Therefore, multiple biomarkers have been investigated for selecting patients who can benefit from immunotherapy.

Tumor mutation burden (TMB) is an emerging biomarker to independently predict response to immunotherapy $(11,12)$. For example, the retrospective analysis of the Checkmate-032 study suggested that the efficacy of nivolumab combined with ipilimumab was improved in patients with high TMB compared with in those with low TMB (ORR, 46.2 vs. 22.2\%) (11). Currently, the evaluation of TMB is based on expensive, large next-generation sequencing (NGS) gene panels or whole-exome sequencing. Several studies have demonstrated that single gene mutations, such as driver mutations in polymerase $\varepsilon$ catalytic subunit A (POLE)/polymerase $\delta$ catalytic subunit gene 1 (POLD1) genes and pathogenetic mutations in mismatch repair genes, are associated with high TMB, which may provide an economical, simple and complementary method for predicting TMB and response to immunotherapy (13-15). However, the aforementioned studies mainly focused on colorectal and endometrial carcinoma. The molecular characteristics that may contribute to high TMB in lung cancer have not been fully documented. Therefore, in the present study, a retrospective, cohort study was conducted to comprehensively investigate the clinicopathological and molecular features of patients with lung cancer with extremely high mutation burden (hypermutation).

In addition to TMB, other molecular biomarkers have been identified to affect patient response to immunotherapy. High levels of microsatellite instability (MSI), deficient mismatch repair and PD-L1 expression have been approved by the Food and Drug Administration (FDA) as predictive biomarkers of immunotherapy across multiple types of cancer, such as NSCLC, triple-negative breast cancer and gastric or gastroesophageal junction adenocarcinoma (16-19). Additionally, alterations in DNA damage response (DDR) genes may be associated with high TMB and improved clinical benefits from immunotherapy in patients with NSCLC (20). Tumors with co-occurring TP53/KRAS gene mutations exhibited remarkable clinical benefit from immunotherapy with PD-1 inhibitors (21). However, some driver mutations in NSCLC tend to predict a poorer response to immunotherapy, such as EGFR sensitive mutations and ALK fusions (22). Somatic mutations in PTEN, $\beta$-2 microglobulin (B2M), serine-threonine kinase 11 (STK11), Kelch-like ECH-associated protein 1 (KEAP1), murine double minute 2 (MDM2) and 11q13 amplification have also been negatively associated with immunotherapy response (23-27). The current study aimed to explore the prevalence of these immunotherapy-associated biomarkers in a hypermutant lung cancer cohort.

\section{Materials and methods}

Patient samples. A total of 1,000 patients with lung cancer who underwent genetic testing using the NGS technology at
Geneplus-Beijing (Beijing, China) between November 2017 and September 2019 were retrospectively enrolled in the present study, irrespective of the type of prior treatment and treatment response. The clinical characteristics of the patients are summarized in Table I. Fresh tissues or tissues fixed using $10 \%$ formalin at room temperature for $24 \mathrm{~h}$ and embedded in paraffin [formalin-fixed paraffin-embedded (FFPE) tissues], including those prepared from pleural effusions, and $10 \mathrm{ml}$ matched peripheral blood were obtained from each patient to perform a matched tumor-normal NGS testing. All procedures were conducted in accordance with the Declaration of Helsinki and written informed consent was provided by all participants.

Sequencing and analysis. Comprehensive genomic profiling was performed using a custom-designed NGS panel containing 1,021 cancer-associated genes. The captured genomic regions included the most common driver genes of solid tumors, including lung cancer. Sample processing, DNA extraction, library preparation, target capture, sequencing and bioinformatic analysis were conducted as previously described $(28,29)$. Briefly, the genomic DNA from white blood cells isolated from peripheral blood, fresh tissues and FFPE samples was extracted using the CWE9600 Blood DNA kit (CoWin Biosciences) and the Maxwell ${ }^{\circledR} 16$ FFPE Plus LEV DNA Purification kit (Promega Corporation) according to the manufacturer's protocol. Illumina TruSeq DNA Library Preparation kits (Illumina, Inc.) were utilized to prepare the sequencing libraries. The libraries were then hybridized to custom-designed biotinylated oligonucleotide probes (Roche NimbleGen, Inc.) targeting 1,021 genes, including 14 genes with therapeutic value, recommended by the National Comprehensive Cancer Network guidelines (30-34) or approved by the FDA, and 220, 98 and 689 genes with therapeutic, diagnostic or prognostic value based on well-powered studies, multiple small studies, and small studies or case reports, respectively (Table SI). The prepared libraries were sequenced on the NextSeq CN 500 system (Illumina, Inc.), according to the manufacturer's instructions. The calling of somatic single nucleotide variants (SNVs) and small insertions and deletions (InDels) was executed using the MuTect (v1.1.4) and GATK (v3.4-46-gbc02625) softwares (both Broad Institute). Copy number variations and structural variations were identified using Contra (v2.0.8) (35) and NoahCare structural variations detection (in house), respectively. TMB was calculated as the number of somatic non-synonymous SNVs and InDels per $\mathrm{Mb}$ in the coding region, with a variant allele fraction of $\geq 0.03$. The threshold of hypermutation was determined using the following formula, previously used by Zehir et al (36) from Memorial Sloan Kettering Cancer Center: Median (TMB) $+2 \mathrm{x}$ IQR (TMB), where IQR represents the interquartile range. The MSI status was inferred using the MSIsensor (v0.5) software (37) and MSI-high was defined on an empirically defined cut-off of MSI score $>8 \%$. SNVs and InDels, copy number variants, structural variants and TMB and MSI are provided in Tables SII, SIII, SIV and SV, respectively.

Differentially mutated genes (DMGs) and pathway enrichment analysis. The DMGs between cohorts were identified using two-tailed Fisher's exact test. $\mathrm{P} \leq 0.05$ was considered to indicate a statistically significant difference. Kyoto Encyclopedia of Genes and Genomes (KEGG) analysis was performed using the Web-based Gene Set Analysis Toolkit (WebGestalt; 
Table I. Comparison of clinicopathological characteristics between patients with hypermutant (n=67) and non-hypermutant ( $\mathrm{n}=933)$ lung cancer.

\begin{tabular}{|c|c|c|c|}
\hline Characteristics & Hypermutant cohort & Non-hypermutant cohort & P-value \\
\hline Median age at diagnosis (range), years & $62(43-79)$ & $61(18-94)$ & 0.0198 \\
\hline $\operatorname{Sex}, \mathrm{n}(\%)$ & & & $<0.0001$ \\
\hline Male & $58(86.6)$ & $552(59.2)$ & \\
\hline Female & $9(13.4)$ & $381(40.8)$ & \\
\hline Histological subtype, n (\%) & & & 0.0215 \\
\hline Adenocarcinoma & $42(62.7)$ & $742(79.5)$ & \\
\hline Squamous & $15(22.4)$ & $122(13.1)$ & \\
\hline SCLC & $4(6.0)$ & 24 (2.6) & \\
\hline Other & $2(3.0)$ & 15 (1.6) & \\
\hline N/A & $4(6.0)$ & $30 \quad(3.2)$ & \\
\hline Clinical stage, n (\%) & & & 0.5087 \\
\hline $\mathrm{I} / \mathrm{II}$ & $4(6.0)$ & $105(11.3)$ & \\
\hline III & $6 \quad(9.0)$ & $75(8.0)$ & \\
\hline IV & $26(38.8)$ & $399(42.8)$ & \\
\hline N/A & $31(46.3)$ & $354(37.9)$ & \\
\hline Smoking history, n (\%) & & & $<0.0001$ \\
\hline Never smokers & $10(14.9)$ & $498(53.4)$ & \\
\hline Smokers & $57(85.1)$ & $435(46.6)$ & \\
\hline Previous treatment, n (\%) & & & 0.6062 \\
\hline No & $48(71.6)$ & $695(74.5)$ & \\
\hline Yes & $19(28.4)$ & $238(25.5)$ & \\
\hline Family history, n (\%) & & & 0.5237 \\
\hline Yes & $13(19.4)$ & $151(16.2)$ & \\
\hline No & $39(58.2)$ & $560(60.0)$ & \\
\hline $\mathrm{N} / \mathrm{A}$ & $15(22.4)$ & $222(23.8)$ & \\
\hline Sample type, n (\%) & & & 0.1404 \\
\hline FFPE tissue & $61(91.0)$ & $787(84.4)$ & \\
\hline Fresh tissue & $6 \quad(9.0)$ & $146(15.6)$ & \\
\hline
\end{tabular}

P-values were calculated using the Mann-Whitney $U$ test or $\chi^{2}$ test. $\mathrm{P} \leq 0.05$ was considered to indicate a statistically significant difference. Missing information in histological subtype, clinical stage and family history was not included in these statistical analyses. SCLC, small cell lung cancer; N/A, not available; FFPE, formalin-fixed, paraffin-embedded.

http://www.webgestalt.org/) to explore whether the DMGs that were more frequently mutated in the hypermutant cohort were enriched in certain signaling pathways. The significance of mutation enrichment was determined by applying a hypergeometric test and was adjusted for multiple testing using the Benjamini-Hochberg false discovery rate.

Statistical analysis. The somatic mutation profiles in The Cancer Genome Atlas (TCGA) database from 1,031 NSCLC samples, including 562 patients with adenocarcinoma and 469 patients with squamous cell cancer, were downloaded from CBioPortal (https://www.cbioportal.org). The difference in age at diagnosis between the cohorts were evaluated using a two-tailed unpaired Mann-Whitney U test. $\chi^{2}$ test was utilized to assess the differences in other demographic characteristics. Missing data regarding histological subtype, clinical stage and family history were not included in the statistical analyses. The correlation between sex and smoking history was examined using Spearman's rank correlation analysis. Cox multivariate regression analysis was used to further verify the association between clinical characteristics and TMB. $\mathrm{P} \leq 0.05$ was considered to indicate a statistically significant difference. Statistical analyses were performed using GraphPad Prism 8.0.2 (GraphPad Software, Inc.) or SPSS v19 software (IBM Corp.).

\section{Results}

Study design and patient demographics. The flow chart of the methodology of the present study is presented in Fig. 1A. To determine the threshold of hypermutation in patients with lung cancer, the TMB status of 1,000 selected patients with lung cancer was screened. The distribution of TMB is presented in Fig. 1B. Among these patients, the median TMB was 5 muts/Mb (range, $0-80$ muts/Mb). TMB of 17.24 muts/Mb was 
A

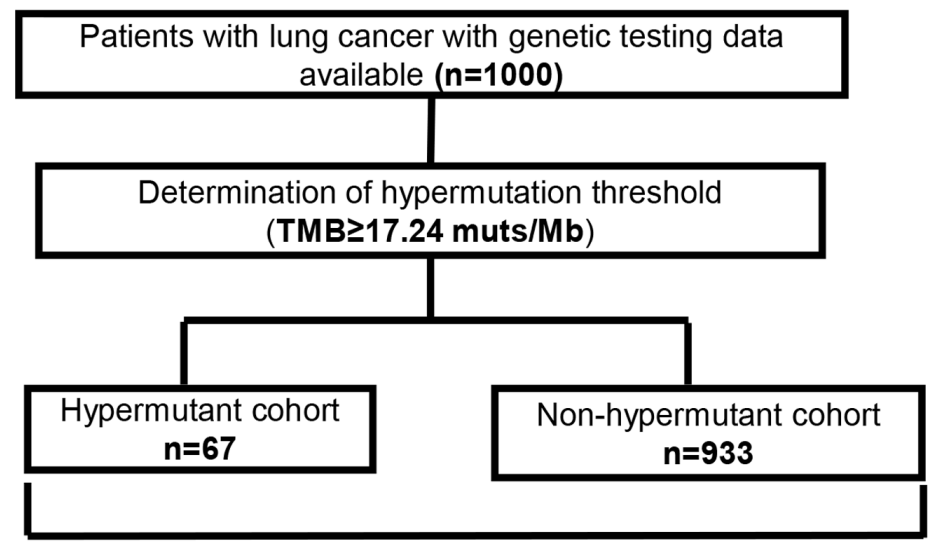

Clinical and molecular features comparison

B

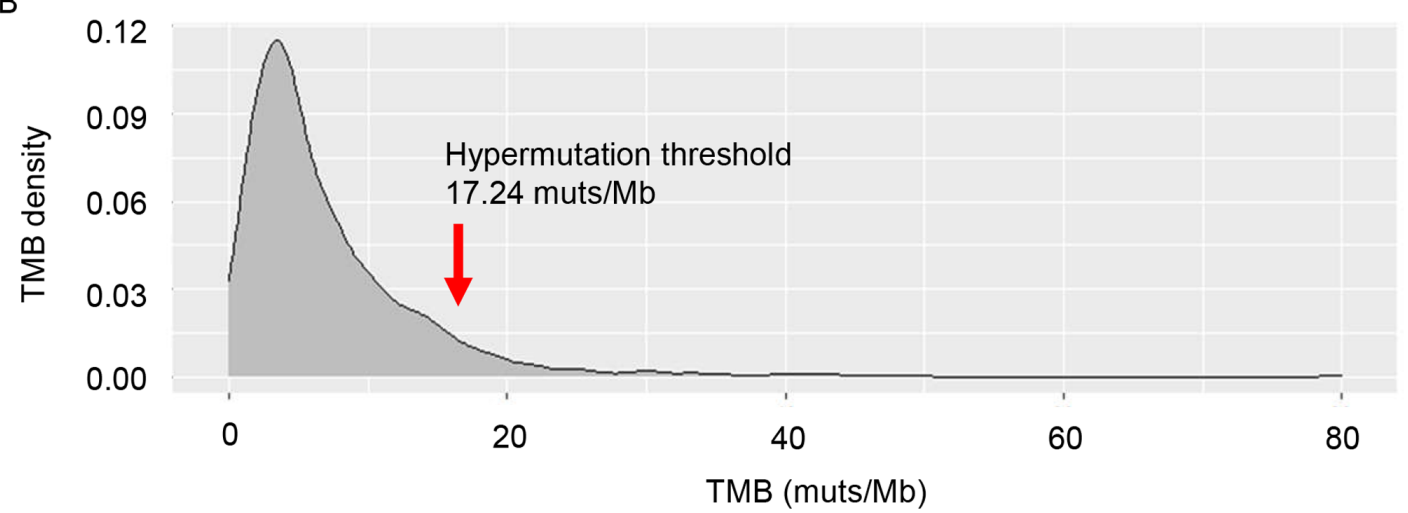

Figure 1. Study design and TMB distribution. (A) Flow chart of the methodology used in the present study. (B) TMB distribution of 1,000 patients with lung cancer. TMB, tumor mutation burden. TMB, tumor mutation burden; muts, mutations.

defined as the threshold according to the criterion for hypermutation set in the current study. Consequently, 67 patients were considered to be hypermutant, and the remaining patients were non-hypermutant $(n=933)$. The median TMB for the hypermutant and non-hypermutant cohorts was 22 (range, 17.28-80 muts/Mb) and 4.8 muts/Mb (range, 0-17 muts/Mb), respectively.

The clinicopathological characteristics of patients are summarized in Table I. The proportion of males (86.6 vs. 59.2\%) and smokers (85.1 vs. $46.6 \%$ ) was higher in the hypermutant cohort compared with in the non-hypermutant cohort (both $\mathrm{P}<0.0001)$. In addition, a strong correlation between smoking and sex (male smokers vs. female smokers, 79.5 vs. 9.1\%; Spearman rank correlation, 0.659; 95\% CI, 0.551-0.748) was revealed in the present study (data not shown). Additionally, compared with in the non-hypermutant cohort, the proportion of patients with squamous cell carcinoma and SCLC was higher in the hypermutant cohort (22.4 vs. $13.1 \%$ and 6.0 vs. $2.6 \%$, respectively; $\mathrm{P}=0.0215)$. In addition, the age at diagnosis of hypermutant patients was significantly increased compared with that of non-hypermutant patients $(\mathrm{P}=0.0198)$. Cox multivariate regression analyses further confirmed that TMB was associated with sex, smoking history and histological subtype, but not with age at diagnosis (Table SVI).

Mutation profiles of hypermutant and non-hypermutant lung cancer. The somatic mutation profiles of hypermutant and non-hypermutant samples are presented in Fig. 2 (top 25 genes for each group). As shown in Fig. 2, the occurrence of mutations in multiple genes was higher in the hypermutant compared with in the non-hypermutant cohort. TP53 was the most frequently mutated gene in both groups, with a mutation frequency of 89.6 and $59.9 \%$ in the hypermutant and non-hypermutant group, respectively $(\mathrm{P}<0.001)$. Low-density lipoprotein receptor-related protein 1B (LRP1B) exerted the most significant difference on mutation rate between the hypermutant and non-hypermutant cohorts (67.2 vs. 14.3\%; $\mathrm{P}<0.001)$. In addition, $51.1 \%$ of cases with LRP1B mutations in the hypermutant cohort harbored $>2$ mutations, which was significantly higher compared with in the non-hypermutant cohort (21.8\%; $\mathrm{P}=0.0002)$ (data not shown). Only EGFR mutations were more frequently observed in the non-hypermutant cohort compared with in the hypermutant cohort (48.9 vs. $22.4 \%$; $\mathrm{P}<0.001$ ).

Subsequently, the mutational profiles of common driver genes in NSCLC were compared (Fig. 3). In the hypermutant cohort (Fig. 3A), the most frequently mutated genes were EGFR and KRAS, with a mutation rate of 18 and $19 \%$, respectively. EGFR sensitive mutations (exon 19 deletions, Leu858Arg and other missense mutations that are sensitive to first- and second-generation EGFR tyrosine kinase inhibitors) and amplification were identified in only 7.5 and $9 \%$ of hypermutant patients, respectively. BRAF non-V600E mutations were found in 4 cases in the hypermutant cohort. In the 


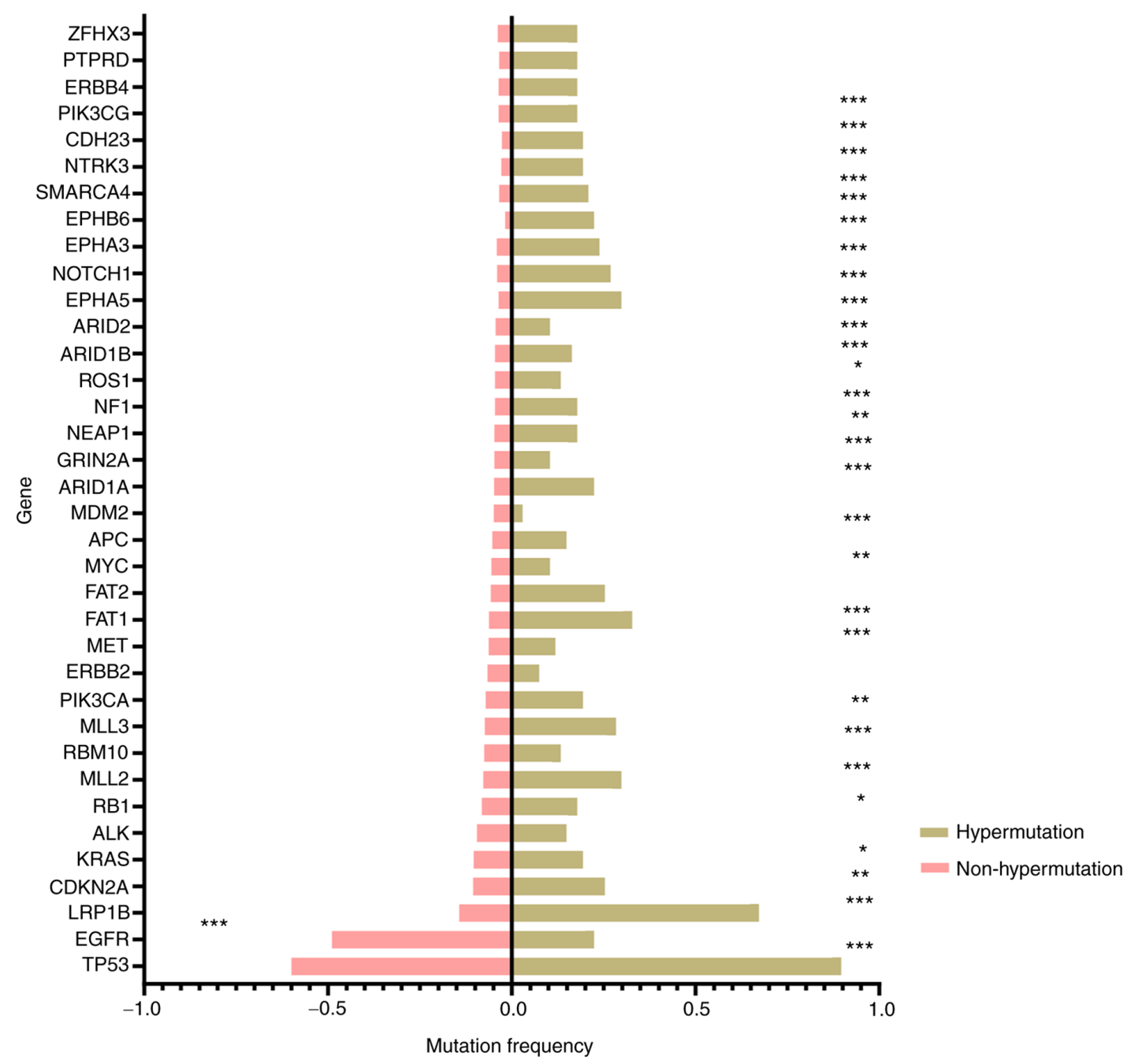

Figure 2. Comparison of mutation frequency in patients with hypermutant and non-hypermutant lung cancer (top 25 genes). Two-tailed Fisher's exact test was used to evaluate whether significant differences were obtained in the mutation frequency of each gene between cohorts. ${ }^{*} \mathrm{P}<0.05 ;{ }^{* * *} \mathrm{P}<0.01 ;{ }^{* * *} \mathrm{P}<0.001$.

non-hypermutant cohort (Fig. 3B), EGFR mutations were detected in $48 \%$ of cases. Furthermore, EGFR sensitive mutations were identified in $43.2 \%$ of non-hypermutant patients, and among them, 45 cases harbored EGFR secondary resistance mutations. EGFR amplification was found in $12.2 \%$ of non-hypermutant patients, with $76.3 \%$ of cases accompanied with EGFR sensitive mutations. Compared with in the hypermutant cohort, fewer patients in the non-hypermutant cohort harbored KRAS mutations (10\%). ALK, ROS1 and RET rearrangements were only observed in the non-hypermutant cohort with a frequency of $6,2.3$ and $1.3 \%$, respectively. Finally, BRAF mutations were detected in 23 cases, including $11 \mathrm{~V} 600 \mathrm{E}$ and 12 non-V600E mutations, representing $2.5 \%$ of all patients in the non-hypermutant cohort.

Molecular features associated with immunotherapy efficacy. The genetic factors associated with immunotherapy efficacy in the hypermutant cohort were analyzed. The results revealed that MSI-high was observed in 6 cases in the hypermutant cohort $(9.0 \%)$ and only 1 patient in the non-hypermutant cohort $(0.1 \%)$ (data not shown). Subsequently, the prevalence of DDR variants $(20,38,39)$ (Table SVII) between the hypermutant and non-hypermutant cohorts was compared. A total of 105 mutations were identified in DDR genes in $70.1 \%$ of patients in the hypermutant cohort, while 321 mutations in DDR genes were detected in $27.0 \%$ of patients in the non-hypermutant cohort (data not shown). Mutations in multiple DDR genes were enriched in the hypermutant cohort, including mutations in ATM, POLE/POLD1 and BRCA1/2 genes (Fig. 4A). As shown in Fig. 4B and C, 16 mutations (including one copy number variant) were detected in POLE/POLD1 genes. According to the criteria for driver mutations proposed by Campbell et al (40), no known driver mutations were detected in POLE/POLD1 genes. In addition, several mutations in POLD1 (E795Q and S816C) and POLE (A1528T and P1205L) genes were considered as non-driver mutations, according to the POLE/POLD1 variants and associated mutation burden referred by Campbell et al (40). The function of other variants remains to be characterized. TP53 and KRAS co-alterations were identified in $16.4 \%$ of patients in the hypermutant cohort, and significantly fewer in the non-hypermutant cohort (5.8\%; $\mathrm{P}=0.002$; data not shown). Furthermore, several genetic alterations were negatively associated with response to immunotherapy, including three PTEN loss-of-function (LOF) mutations, two B2M LOF mutations, five EGFR sensitive mutations, three STK11 LOF mutations, 


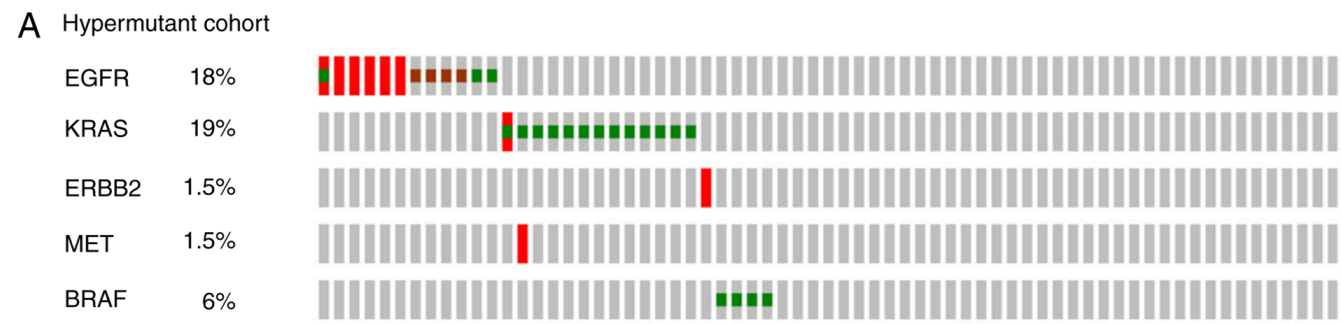

B Non-hypermutant cohort

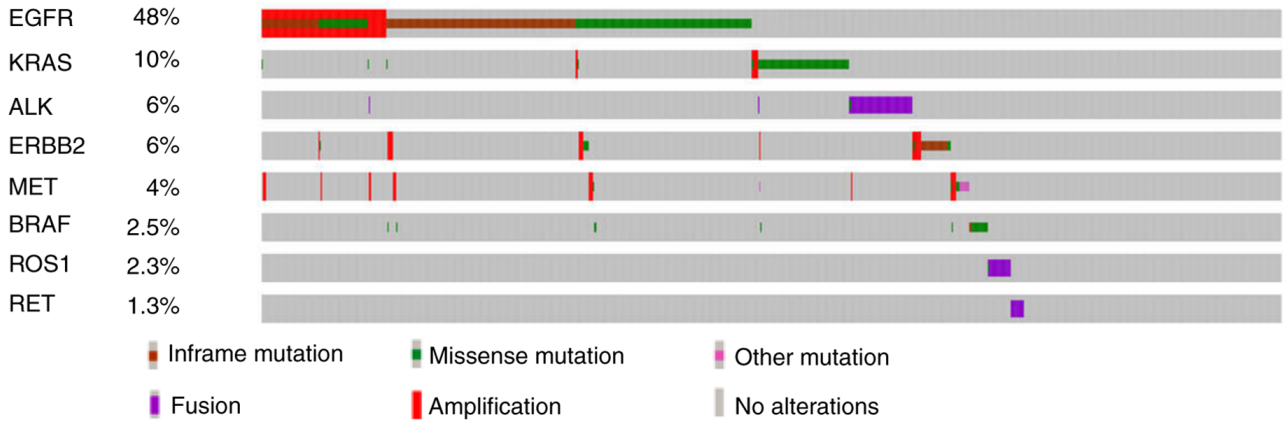

Figure 3. Somatic mutational profiles of common driver genes in patients with hypermutant and non-hypermutant non-small cell lung cancer. (A) Hypermutant cohort and (B) non-hypermutant cohort.

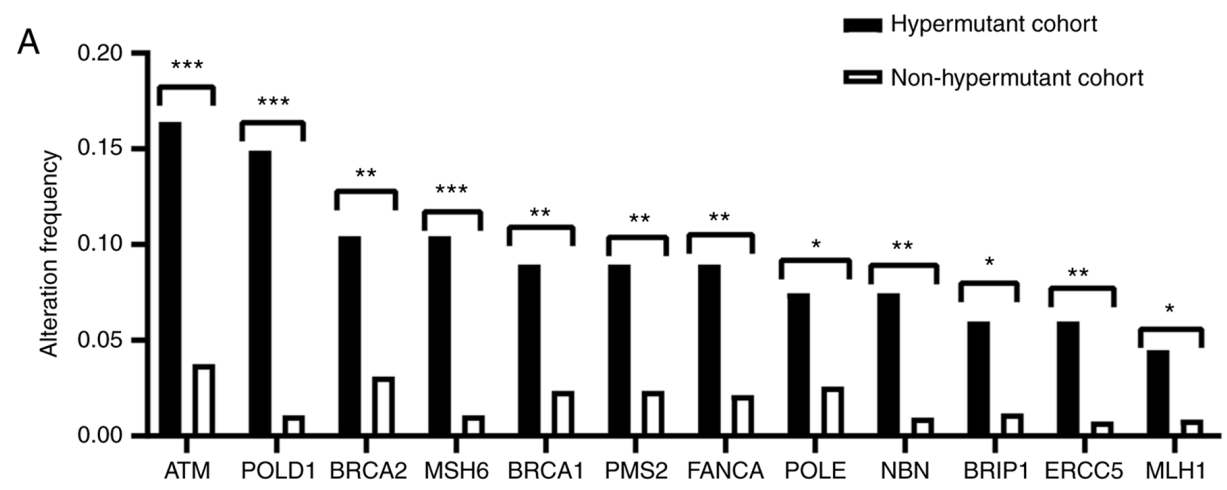

B

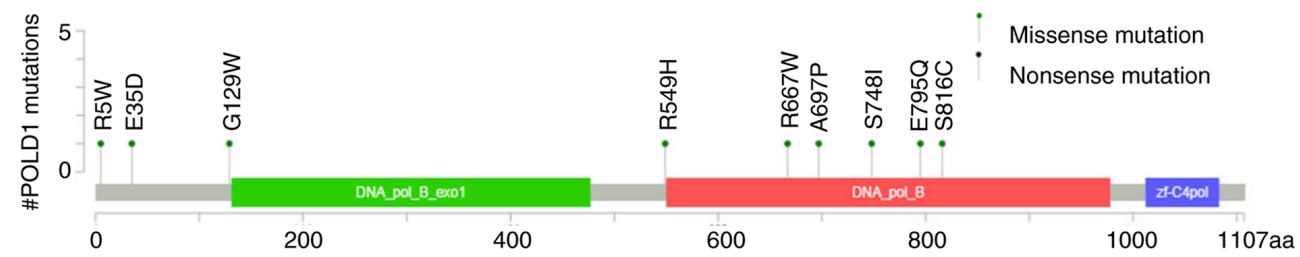

C

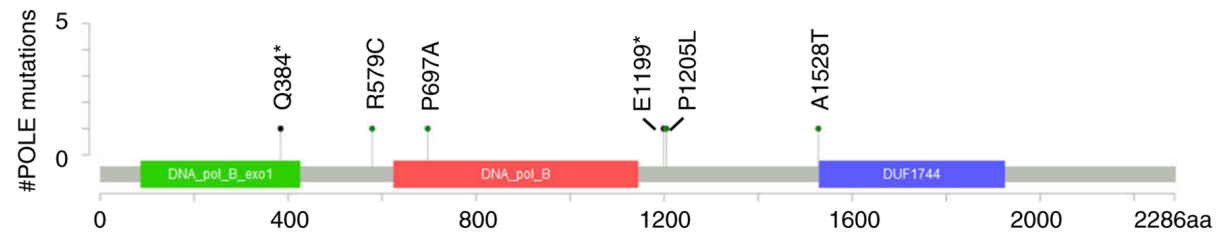

Figure 4. Mutational features of DDR genes. (A) Alterations in DDR genes with significant differences between hypermutant and non-hypermutant cohorts. Differences were examined using a Fisher's exact test. ${ }^{*} \mathrm{P}<0.05 ;{ }^{* *} \mathrm{P}<0.01 ;{ }^{* * * *} \mathrm{P}<0.001$. Mutations in (B) POLD1 and (C) POLE genes. DDR, DNA damage repair; POLD1, polymerase $\delta$ catalytic subunit gene 1 ; POLE, polymerase $\varepsilon$ catalytic subunit A.

one KEAP1 LOF mutation, two cases with 11q13 amplification (CCND1, FGF3, FGF4 and FGF19) and one case with MDM2 amplification (data not shown).
Identification of DMGs and enrichment analysis. To identify genes with significantly higher alteration frequency in the hypermutant group compared with in the non-hypermutant 
A

MicroRNAs in cancer

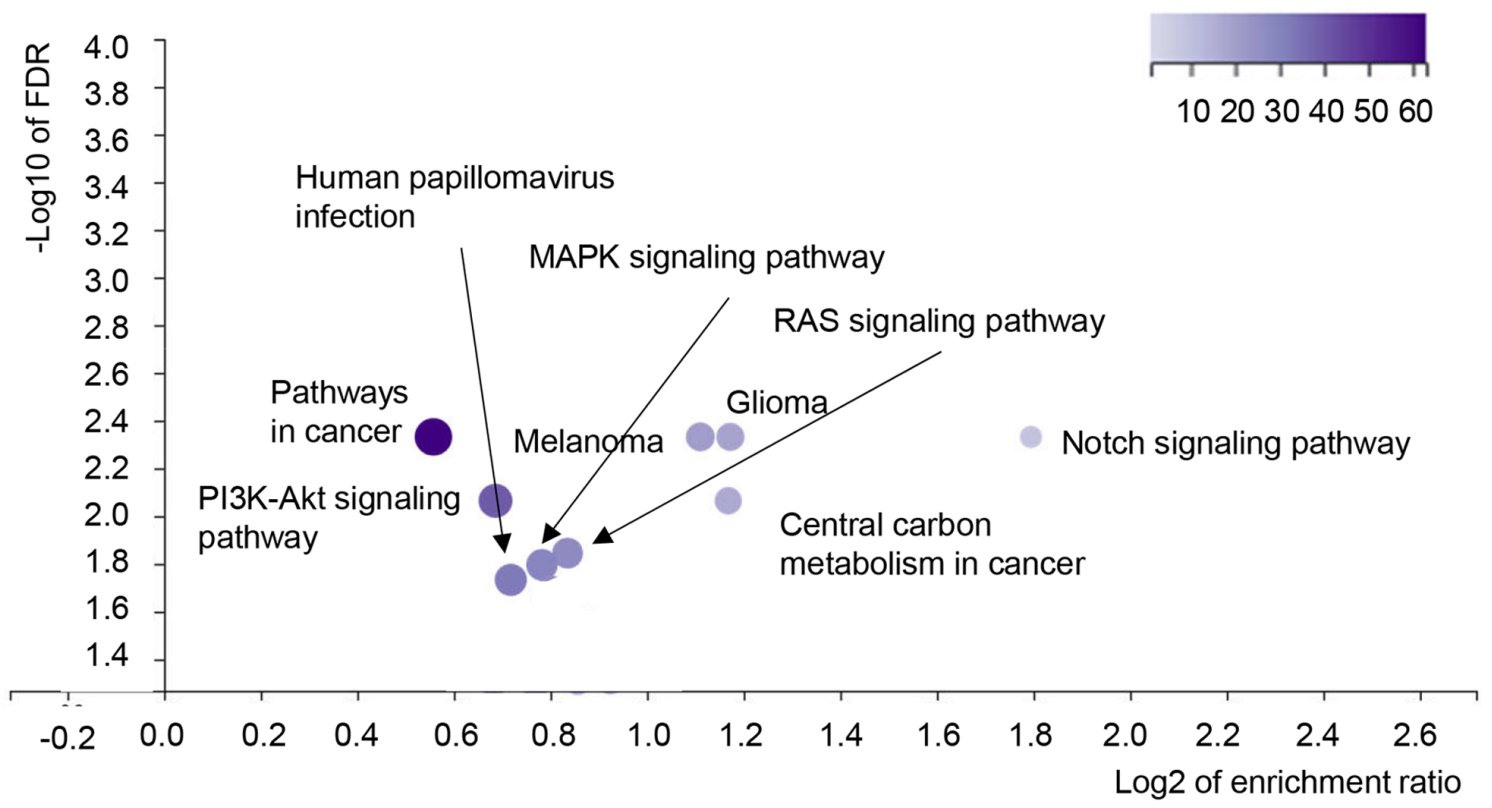

B

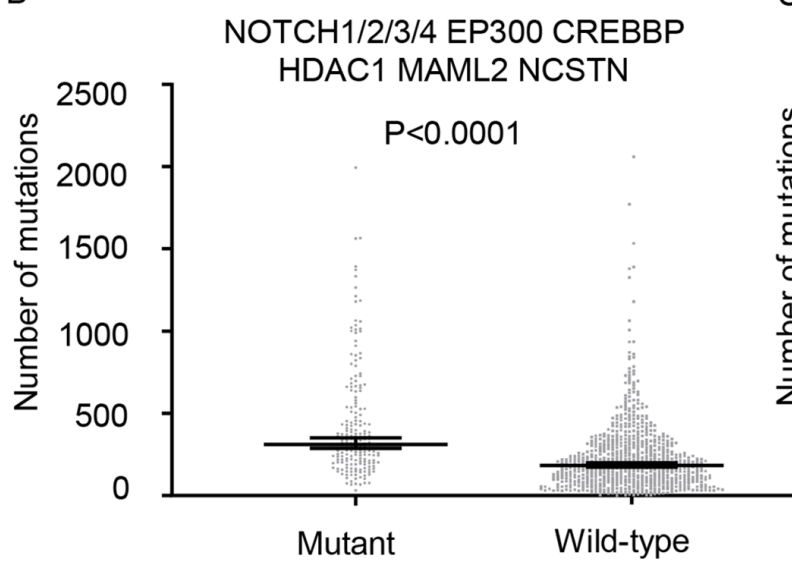

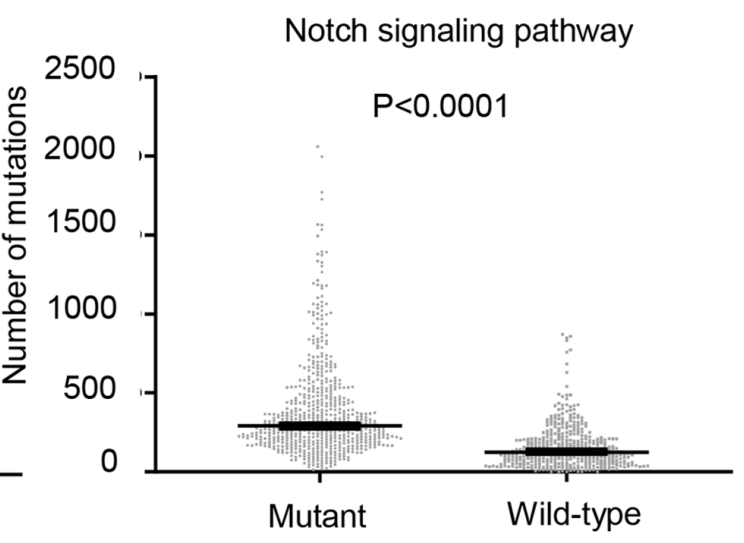

Figure 5. Identification and validation of Notch signaling mutation enrichment in the hypermutant cohort. (A) Top 10 pathways enriched in the hypermutant cohort. (B) Validation of nine genes involved in the enriched Notch signaling pathway using the mutation profiles of NSCLC downloaded from TCGA database. (C) Validation of genes involved in the entire Notch signaling using the mutation profiles of NSCLC downloaded from TCGA database. Two-tailed unpaired Mann-Whitney U test was used to compare the number of mutations between Notch signaling mutant and wild-type cohorts. NSCLC, non-small cell lung cancer; TCGA, The Cancer Genome Atlas; FDR, false discovery rate; CREBBP, CREB-binding protein; EP300, E1A binding protein P300; MAML2, mastermind-like transcriptional coactivator 2; NCSTN, nicastrin; HDAC1, histone deacetylase 1.

cohort, Fisher's exact test was performed. The DMGs enriched in the hypermutant cohort are summarized in Table SVIII. Webgestalt was used for the enrichment analysis. The top 10 pathways are shown in Fig. 5A, including the Notch signaling pathway, MAPK signaling pathway and RAS signaling pathway. Mutations in the Notch signaling pathway were enriched in the hypermutant cohort, with an enrichment ratio of 3.49. This signaling pathway included NOTCH1/2/3/4, CREB-binding protein (CREBBP), E1A binding protein P300 (EP300), mastermind-like transcriptional coactivator 2 (MAML2), nicastrin (NCSTN) and histone deacetylase 1 (HDAC1); these genes were completely mutated in $64.2 \%$ of cases in the hypermutant cohort (data not shown). To validate the enrichment of mutations in the Notch signaling pathway in the hypermutant cohort, lung adenocarcinoma and squamous cell carcinoma data from TCGA database were analyzed $(n=1,031)$. Patients with mutations in these nine genes exhibited more non-synonymous mutations in the coding regions compared with those without these mutations (median, 310 vs. 183.5; P<0.0001; Fig. 5B). Similarly, when the validated genes were expanded to all the genes involved in the Notch signaling pathway (41) (ARRDC1, CNTN6, CREBBP, EP300, HES1, HES2, HES3, HES4, HES5, HEY1, HEY2, HEYL, KAT2B, KDM5A, NOTCH1, NOTCH2, NOTCH3, NOTCH4, NOV, NRARP, PSEN2, LFNG, ITCH, NCSTN, SPEN, JAG1, APH1A, FBXW7, FHL1, THBS2, HDAC2, MFAP2, CUL1, RFNG, NCOR1, NCOR2, MFAP5, HDAC1, NUMB, JAG2, MAML3, MFNG, CIR1, CNTN1, MAML1, MAML2, NUMBL, PSEN1, PSENEN, RBPJ, RBPJL, RBX1, SAP30, SKP1, SNW1, CTBP1, CTBP2, ADAM10, APH1B, ADAM17, DLK1, DLL1, DLL3, DLL4, DNER, DTX1, DTX2, DTX3, DTX3L, DTX4, EGFL7), a 
significant difference was also observed (median, 292 vs. 125; P<0.0001; Fig. 5B).

\section{Discussion}

It has been reported that hypermutation is frequently found in melanoma, lung and bladder cancer (42-44). The present retrospective cohort study comprehensively explored the clinicopathological and molecular features of patients with hypermutant lung cancer. The present study may provide important insights into hypermutant lung cancer among the Chinese population. To the best of our knowledge, the present study was the first to comprehensively investigate the clinical and genetic characteristics of hypermutant lung cancer in a Chinese population.

First, the clinical characteristics between cohorts were compared, and the results revealed that hypermutation was more frequently observed in certain groups of patients, including males, smokers and specific histological subtypes. A previous study investigated the association between smoking and mutational burden. The results demonstrated that the total number of point mutations in the coding regions was higher in smokers (median, 209; range, 104-1,363) compared with non-smokers (median, 18; range, 10-22) (43). Other studies also obtained similar conclusions $(45,46)$. Additionally, the present findings were consistent with the aforementioned studies. In addition, the strong correlation between smoking and sex observed in the present study may be associated with smoking habits in the Chinese population. In China, the smoking prevalence was $47.2 \%$ (range, $46.9-47.5 \%$ ) for men and 2.7\% (range, 2.6-2.8\%) for women in 2013 (47). Therefore, the present study hypothesized that the significantly higher rate of males in the hypermutant cohort may result from the higher number of smokers in this cohort. The current findings were consistent with a previous study demonstrating higher TMB in squamous carcinoma and SCLC compared with in adenocarcinoma (48).

Subsequently, the mutation spectra of both cohorts were analyzed. LRP1B was more frequently mutated in the hypermutant cohort. LRP1B encodes a member of the low-density lipoprotein receptor family. These receptors serve a wide variety of roles in normal cell function and development by interacting with multiple ligands (49). Several studies have demonstrated an association between LRP1B mutations and a high level of TMB in patients with NSCLC and melanoma; these studies suggested that in patients with LRP1B mutations, immune response and cell cycle regulation circuits were among the top enriched pathways $(50,51)$. Although the mechanism underlying the association between TMB and LRP1B is not entirely clear, the current study supported the aforementioned findings. The distribution of KRAS and EGFR mutations identified in the present study was also consistent with previous studies, suggesting the positive effect of KRAS mutations and negative effect of EGFR mutations on immunotherapy response (21,52). ALK, ROS1 and RET rearrangements were only found in the non-hypermutant cohort, which may suggest a negative association with hypermutation, thereby affecting the response to immunotherapy (53).

The genetic factors affecting immunotherapy response were also analyzed. MSI-high was reported in $0.2 \%$ of patients with non-squamous NSCLC (54). In the present study, the prevalence of MSI-high was extremely high in the hypermutant cohort (9\%), indicating a potential important role of MSI in hypermutation in lung cancer. The enrichment of DDR gene mutations in the hypermutant cohort suggested that mutations in DDR genes may serve as biomarkers for predicting TMB and patient response to immunotherapy. A previous study has confirmed elevated TMB and improved efficacy of immunotherapy in patients with pathogenic DDR alterations (20). Co-mutations of KRAS and TP53 were more frequently identified in the hypermutation cohort, supporting the improved clinical outcomes of patients with KRAS and TP53 co-mutations during the period of immunotherapy (21). In addition, several negative biomarkers, such as PTEN mutations and MDM2/4 amplification, in the hypermutant cohort were identified, suggesting hyper-progressive disease or disease resistant to immunotherapy, which should raise concern.

DMGs enriched in the hypermutant cohort were identified in the present study, and an enrichment analysis was performed. The results suggested that mutations in the Notch signaling pathway were associated with high TMB, which was confirmed using molecular profiles of lung cancer in TCGA database. The Notch signaling pathway activates cell proliferation and antagonizes apoptosis, as well as cross-talks with several transcriptional factors to promote epithelial-mesenchymal transition in lung cancer, thus leading to enhanced motility, invasion and metastasis of cancer cells (55). Recently, NOTCH1 has been reported to contribute to an immune-suppressive tumor microenvironment in melanoma (56). Targeting NOTCH1 may therefore affect cell proliferation and survival, and provide an immune-responsive tumor microenvironment, thus improving the efficacy of immunotherapy (56). Another study uncovered a marked association between mutations in NOTCH1/2/3 and improved outcomes in EGFR- and ALK-wild-type patients with NSCLC treated with immune checkpoint inhibitors (57). In addition, deleterious NOTCH mutations exhibited an improved effect compared with non-deleterious mutations (57). However, the underlying mechanism remains unknown. Previous studies found that tumors with Notch family gene (NOTCH1/2/3/4) mutations exerted higher TMBs in multiple types of cancer, including hepatocellular carcinoma, esophageal carcinoma, breast cancer, SCLC, head and neck cancer and cutaneous carcinoma $(58,59)$, which may explain the predictive value of NOTCH mutations to immunotherapy response. In the present study, the strong association between NOTCH gene mutations and high TMB indicated a potential strategy for immunotherapy in patients with lung cancer with mutations in the Notch signaling pathway. However, the mutation types in genes involved in the Notch signaling pathway and the specific genes exhibiting strong predictive value to immunotherapy response should be further investigated.

The FDA has approved FoundationOne ${ }^{\circledR} \mathrm{CDx}$ as the first companion diagnostic to identify patients with solid tumors that are TMB-high $(\geq 10$ muts/Mb) and suitable for treatment with pembrolizumab (60). A previous study conducted by Foundation Medicine described the distribution of TMB across a diverse cohort of 100,000 cases of cancer, with TMB $>20$ muts/Mb designated as high TMB (61). The percentages of 
cases with TMB >20 muts/Mb were 17, 12.3, 11.3 and $9 \%$ for NSCLC (not otherwise specified) $(n=2,636)$, lung adenocarcinoma $(n=11,855)$, lung squamous cell carcinoma $(n=2,102)$ and lung small cell undifferentiated carcinoma $(n=913)$, respectively (61). However, in the present study, hypermutant patients (TMB $\geq 17.24$ muts/Mb) constituted $6.7 \%$ of the whole population. TMB cut-offs may differ depending on sample type, tumor type, patient subgroup, therapy investigated and assay used. More specifically, the capture region, number of genes, sequencing depth and TMB calculation method of a panel may affect the TMB threshold. Despite the difference between TMB cut-offs and sequencing details, the findings of the present study may be valuable thanks to the scientific method for TMB cut-off determination and controlled study design.

However, there were a few limitations due to the retrospective nature of the present study. Information on whether the patients received immunotherapy after genetic testing and corresponding treatment response was not available. The efficacy of immune checkpoint inhibitors in the hypermutant cohort was of great value and could be further researched. In addition, $\sim 78 \%$ of patients in the current study were diagnosed with adenocarcinoma. It may be interesting to explore TMB-associated molecular characteristics in adenocarcinoma only in a Chinese population.

In conclusion, the present cohort study suggested that hypermutant lung cancer exhibited distinctive genetic profiles, including high occurrence of MSI-high, high frequency of mutations in DDR genes and genes involved in the Notch signaling pathway, which may be associated with high levels of TMB. In addition, patients with hypermutant lung cancer may be more likely to have a history of tobacco use and exhibit the histological subtypes of squamous carcinoma and SCLC. These characteristics may be used as complementary indicators for screening patients sensitive to immunotherapy.

\section{Acknowledgements}

Not applicable.

\section{Funding}

The present study was supported by Government-funded clinical excellence training programme.

\section{Availability of data and materials}

The datasets used and/or analyzed during the current study are available from the corresponding author on reasonable request.

\section{Authors' contributions}

HZ, YW and MZ contributed to conception and design of the study. MZ contributed to the provision of study materials or patients. HZ and MY confirm the authenticity of all the raw data. QJ, HC, RC, XL, JX and MY contributed to acquisition of data. HL, JW, GZ, ET and LZ performed data analysis and interpretation. All authors wrote the manuscript and read and approved the final manuscript.

\section{Ethics approval and consent to participate}

All procedures were in accordance with the 1964 Declaration of Helsinki and its later amendments. The study was performed under a protocol approved by the Institutional Review Board of Geneplus-Beijing. Written informed consent was provided by all participants included in the study.

\section{Patient consent for publication}

Not applicable.

\section{Competing interests}

MY and RC are employees of Geneplus-Beijing. All other authors declare that they have no competing interests.

\section{References}

1. Chen W, Zheng R, Baade PD, Zhang S, Zeng H, Bray F, Jemal A, $\mathrm{Yu}$ XQ and He J: Cancer statistics in China, 2015. CA Cancer J Clin 66: 115-132, 2016.

2. Chung HC, Piha-Paul SA, Lopez-Martin J, Schellens JHM, Kao S, Miller WH Jr, Delord JP, Gao B, Planchard D, Gottfried M, et al: Pembrolizumab after two or more lines of prior therapy in patients with advanced small-cell lung cancer (SCLC): Results from the KEYNOTE-028 and KEYNOTE-158 studies. Cancer Res 79: CT073, 2019.

3. Horn L, Mansfield AS, Szczesna A, Havel L, Krzakowski M, Hochmair MJ, Huemer F, Losonczy G, Johnson ML, Nishio M, et al: First-line atezolizumab plus chemotherapy in extensive-stage small-cell lung cancer. N Engl J Med 379: 2220-2229, 2018 .

4. Reck M, Rodriguez-Abreu D, Robinson AG, Hui R, Csőszi T, Fülöp A, Gottfried M, Peled N, Tafreshi A, Cuffe S, et al: Pembrolizumab versus chemotherapy for PD-L1-positive non-small-cell lung cancer. N Engl J Med 375: 1823-1833, 2016.

5. Herbst RS, Morgensztern D and Boshoff C: The biology and management of non-small cell lung cancer. Nature 553: 446-454, 2018.

6. Antonia SJ, López-Martin JA, Bendell J, Ott PA, Taylor M, Eder JP, Jäger D, Pietanza MC, Le DT, de Braud F, et al: Nivolumab alone and nivolumab plus ipilimumab in recurrent small-cell lung cancer (CheckMate 032): A multicentre, open-label, phase 1/2 trial. Lancet Oncol 17: 883-895, 2016.

7. Brahmer J, Reckamp KL, Baas P, Crinò L, Eberhardt WE, Poddubskaya E, Antonia S, Pluzanski A, Vokes EE, Holgado E, et al: Nivolumab versus docetaxel in advanced squamous-cell non-small-cell lung cancer. N Engl J Med 373: 123-135, 2015.

8. Borghaei H, Paz-Ares L, Horn L, Spigel DR, Steins M, Ready NE, Chow LQ, Vokes EE, Felip E, Holgado E, et al: Nivolumab versus docetaxel in advanced nonsquamous non-small-cell lung cancer. N Engl J Med 373: 1627-1639, 2015.

9. Rittmeyer A, Barlesi F, Waterkamp D, Park K, Ciardiello F, von Pawel J, Gadgeel SM, Hida T, Kowalski DM, Dols MC, et al: Atezolizumab versus docetaxel in patients with previously treated non-small-cell lung cancer (OAK): A phase 3, open-label, multicentre randomised controlled trial. Lancet 389: 255-265, 2017

10. Herbst RS, Baas P, Kim DW, Felip E, Pérez-Gracia JL, Han JY, Molina J, Kim JH, Arvis CD, Ahn MJ, et al: Pembrolizumab versus docetaxel for previously treated, PD-L1-positive, advanced non-small-cell lung cancer (KEYNOTE-010): A randomised controlled trial. Lancet 387: 1540-1550, 2016.

11. Hellmann MD, Callahan MK, Awad MM, Calvo E, Ascierto PA, Atmaca A, Rizvi NA, Hirsch FR, Selvaggi G, Szustakowski JD, et al: Tumor mutational burden and efficacy of nivolumab monotherapy and in combination with ipilimumab in small-cell lung cancer. Cancer Cell 35: 329, 2019.

12. Hellmann MD, Ciuleanu TE, Pluzanski A, Lee JS, Otterson GA, Audigier-Valette C, Minenza E, Linardou H, Burgers S, Salman P, et al: Nivolumab plus ipilimumab in lung cancer with a high tumor mutational burden. N Engl J Med 378: 2093-2104, 2018 . 
13. Wang F, Zhao Q, Wang YN, Jin Y, He MM, Liu ZX and Xu RH: Evaluation of POLE and POLD1 mutations as biomarkers for immunotherapy outcomes across multiple cancer types. JAMA Oncol 5: 1504-1506, 2019.

14. Song Z, Cheng G, Xu C, Wang W, Shao Y and Zhang Y: Clinicopathological characteristics of POLE mutation in patients with non-small-cell lung cancer. Lung Cancer 118: 57-61, 2018.

15. StadlerZK, Battaglin F,MiddhaS,Hechtman JF, TranC,Cercek A, Yaeger R, Segal NH, Varghese AM, Reidy-Lagunes DL, et al Reliable detection of mismatch repair deficiency in colorectal cancers using mutational load in next-generation sequencing panels. J Clin Oncol 34: 2141-2147, 2016.

16. Marcus L, Lemery SJ, Keegan P and Pazdur R: FDA approval summary: Pembrolizumab for the treatment of microsatellite Instability-High solid tumors. Clin Cancer Res 25: 3753-3758, 2019.

17. Narayan P, Wahby S, Gao JJ, Amiri-Kordestani L, Ibrahim A, Bloomquist E, Tang S, Xu Y, Liu J, Fu W, et al: FDA approval summary: Atezolizumab plus paclitaxel protein-bound for the treatment of patients with advanced or metastatic TNBC whose tumors express PD-L1. Clin Cancer Res 26: 2284-2289, 2020.

18. Fashoyin-Aje L, Donoghue M, Chen H, He K, Veeraraghavan J, Goldberg KB, Keegan P, McKee AE and Pazdur R: FDA approval summary: Pembrolizumab for recurrent locally advanced or metastatic gastric or gastroesophageal junction adenocarcinoma expressing PD-L1. Oncologist 24: 103-109, 2019.

19. Sul J, Blumenthal GM, Jiang X, He K, Keegan P and Pazdur R: FDA approval summary: Pembrolizumab for the treatment of patients with metastatic non-small cell lung cancer whose tumors express programmed Death-Ligand 1. Oncologist 21: 643-650, 2016.

20. Ricciuti B, Cheng ML, Recondo G, Nishino M, Umeton R, Sholl LM and Awad MM: DNA damage response gene alterations are associated with high tumor mutational burden and clinical benefit from programmed death 1 axis inhibition in non-small cell lung cancer. J Clin Oncol 37 (Suppl 15): S9077, 2019.

21. Dong ZY, Zhong WZ, Zhang XC, Su J, Xie Z, Liu SY, Tu HY, Chen HJ, Sun YL, Zhou Q, et al: Potential predictive value of TP53 and KRAS mutation status for response to PD-1 blockade immunotherapy in lung adenocarcinoma. Clin Cancer Res 23 3012-3024, 2017.

22. Gainor JF, Shaw AT, Sequist LV, Fu X, Azzoli CG, Piotrowska Z Huynh TG, Zhao L, Fulton L, Schultz KR, et al: EGFR Mutations and ALK rearrangements are associated with low response rates to PD-1 pathway blockade in non-small cell lung cancer: A retrospective analysis. Clin Cancer Res 22: 4585-4593, 2016

23. Peng W, Chen JQ, Liu C, Malu S, Creasy C, Tetzlaff MT, Xu C, McKenzie JA, Zhang C, Liang X, et al: Loss of PTEN promotes resistance to T cell-mediated immunotherapy. Cancer Discov 6 : 202-216, 2016.

24. Sade-Feldman M, Jiao YJ, Chen JH, Rooney MS Barzily-Rokni M, Eliane JP, Bjorgaard SL, Hammond MR, Vitzthum H, Blackmon SM, et al: Resistance to checkpoint blockade therapy through inactivation of antigen presentation. Nat Commun 8: 1136, 2017.

25. Arbour K, Shen R, Plodkowski A, Rizvi H, Ni A, Long N, Halpenny D, Sanchez-Vega F, Rudin C, Riely G and Hellmann M: MA19. 09 concurrent mutations in STK11 and KEAP1 is associated with resistance to PD-(L) 1 blockade in patients with NSCLC despite high TMB. J Thorac Oncol 13 (Suppl 10): S424, 2018.

26. Kato S, Goodman A, Walavalkar V, Barkauskas DA, Sharabi A and Kurzrock R: Hyperprogressors after immunotherapy: Analysis of genomic alterations associated with accelerated growth rate. Clin Cancer Res 23: 4242-4250, 2017.

27. Wang F, Ren C, Zhao Q, Shen L, Dai G, Yuan X, Chen Y, Yang S, Shi J, Hu X, et al: Association of frequent amplification of chromosome 11q13 in esophageal squamous cell cancer with clinical benefit to immune check point blockade. J Clin Oncol 37 (Suppl 15): S4036, 2019.

28. Sun S, Liu Y, Eisfeld AK, Zhen F, Jin S, Gao W, Yu T, Chen L, Wang W, Chen W, et al: Identification of germline mismatch repair gene mutations in lung cancer patients with paired tumor-normal next generation sequencing: A retrospective study. Front Oncol 9: 550,2019.

29. Chen Y, Chen G, Li J, Huang YY, Li Y, Lin J, Chen LZ, Lu JP, Wang YQ, Wang CX, et al: Association of tumor protein $\mathrm{p} 53$ and ataxia-telangiectasia mutated comutation with response to immune checkpoint inhibitors and mortality in patients with non-small cell lung cancer. JAMA Netw Open 2: e1911895, 2019.
30. Benson AB, Venook AP, Al-Hawary MM, Cederquist L, Chen YJ, Ciombor KK, Cohen S, Cooper HS, Deming D, Engstrom PF, et al: NCCN guidelines insights: Colon cancer, version 2.2018. J Natl Compr Canc Netw 16: 359-369, 2018.

31. Ettinger DS, Wood DE, Aggarwal C, Aisner DL, Akerley W, Bauman JR, Bharat A, Bruno DS, Chang JY, Chirieac LR, et al: NCCN guidelines insights: Non-small cell lung cancer, version 1.2020. J Natl Compr Canc Netw 17: 1464-1472, 2019.

32. Haddad RI, Nasr C, Bischoff L, Busaidy NL, Byrd D, Callender G, Dickson P, Duh QY, Ehya H, Goldner W, et al: NCCN guidelines insights: Thyroid carcinoma, version 2.2018. J Natl Compr Canc Netw 16: 1429-1440, 2018.

33. Armstrong DK, Alvarez RD, Bakkum-Gamez JN, Barroilhet L, Behbakht K, Berchuck A, Berek JS, Chen LM, Cristea M, DeRosa M, et al: NCCN guidelines insights: Ovarian cancer, version 1.2019. J Natl Compr Canc Netw 17: 896-909, 2019.

34. Goetz MP, Gradishar WJ, Anderson BO, Abraham J, Aft R, Allison KH, Blair SL, Burstein HJ, Dang C, Elias AD, et al: NCCN guidelines insights: Breast cancer, version 3.2018. J Natl Compr Canc Netw 17: 118-126, 2019.

35. Li J, Lupat R, Amarasinghe KC, Thompson ER, Doyle MA, Ryland GL, Tothill RW, Halgamuge SK, Campbell IG and Gorringe KL: CONTRA: Copy number analysis for targeted resequencing. Bioinformatics 28: 1307-1313, 2012.

36. Zehir A, Benayed R, Shah RH, Syed A, Middha S, Kim HR, Srinivasan P, Gao J, Chakravarty D, Devlin SM, et al: Mutational landscape of metastatic cancer revealed from prospective clinical sequencing of 10,000 patients. Nat Med 23: 703-713, 2017

37. Niu B, Ye K, Zhang Q, Lu C, Xie M, McLellan MD, Wendl MC and Ding L: MSIsensor: Microsatellite instability detection using paired tumor-normal sequence data. Bioinformatics 30 : 1015-1016, 2014

38. Teo MY, Seier K, Ostrovnaya I, Regazzi AM, Kania BE, Moran MM, Cipolla CK, Bluth MJ, Chaim J, Al-Ahmadie H, et al: Alterations in DNA damage response and repair genes as potential marker of clinical benefit from PD-1/PD-L1 blockade in advanced urothelial cancers. J Clin Oncol 36: 1685-1694, 2018.

39. Mehnert JM, Panda A, Zhong H, Hirshfield K, Damare S, Lane K, Sokol L, Stein MN, Rodriguez-Rodriquez L, Kaufman HL, et al: Immune activation and response to pembrolizumab in POLE-mutant endometrial cancer. J Clin Invest 126: 2334-2340, 2016.

40. Campbell BB, Light N, Fabrizio D, Zatzman M, Fuligni F, de Borja R, Davidson S, Edwards M, Elvin JA, Hodel KP, et al: Comprehensive analysis of hypermutation in human cancer. Cell 171: 1042-1056.e10, 2017.

41. Sanchez-Vega F, Mina M, Armenia J, Chatila WK, Luna A, La KC, Dimitriadoy S, Liu DL, Kantheti HS, Saghafinia S, et al: Oncogenic signaling pathways in the cancer genome atlas. Cell 173: 321-337.e10, 2018.

42. Cancer Genome Atlas Network: Genomic classification of cutaneous melanoma. Cell 161: 1681-1696, 2015.

43. Govindan R, Ding L, Griffith M, Subramanian J, Dees ND, Kanchi KL, Maher CA, Fulton R, Fulton L, Wallis J, et al: Genomic landscape of non-small cell lung cancer in smokers and never-smokers. Cell 150: 1121-1134, 2012.

44. Cancer Genome Atlas Research Network: Comprehensive molecular characterization of urothelial bladder carcinoma. Nature 507: 315-322, 2014.

45. Ding L, Getz G, Wheeler DA, Mardis ER, McLellan MD, Cibulskis K, Sougnez C, Greulich H, Muzny DM, Morgan MB, et al: Somatic mutations affect key pathways in lung adenocarcinoma. Nature 455: 1069-1075, 2008.

46. Lee W, Jiang Z, Liu J, Haverty PM, Guan Y, Stinson J, Yue P, Zhang Y, Pant KP, Bhatt D, et al: The mutation spectrum revealed by paired genome sequences from a lung cancer patient. Nature 465: 473-477, 2010.

47. Wang M, Luo X, Xu S, Liu W, Ding F, Zhang X, Wang L, Liu J, Hu J and Wang W: Trends in smoking prevalence and implication for chronic diseases in China: Serial national cross-sectional surveys from 2003 to 2013. Lancet Respir Med 7: 35-45, 2019.

48. Yarchoan M, Hopkins A and Jaffee EM: Tumor mutational burden and response rate to PD-1 inhibition. N Engl J Med 377: 2500-2501, 2017

49. Liu CX, Li Y, Obermoeller-McCormick LM, Schwartz AL and $\mathrm{Bu}$ G: The putative tumor suppressor LRP1B, a novel member of the low density lipoprotein (LDL) receptor family, exhibits both overlapping and distinct properties with the LDL receptor-related protein. J Biol Chem 276: 28889-28896, 2001. 
50. Chen H, Chong W, Wu Q, Yao Y, Mao M and Wang X: Association of LRP1B mutation with tumor mutation burden and outcomes in melanoma and non-small cell lung cancer patients treated with immune check-point blockades. Front Immunol 10: 1113, 2019.

51. Lan S, Li H, Liu Y, Ma L, Liu X, Liu Y, Yan S and Cheng Y: Somatic mutation of LRP1B is associated with tumor mutational burden in patients with lung cancer. Lung Cancer 132: 154-156, 2019.

52. Rizvi H, Sanchez-Vega F, La K, Chatila W, Jonsson P, Halpenny D, Plodkowski A, Long N, Sauter JL, Rekhtman N, et al: Molecular determinants of response to anti-programmed cell death (PD)-1 and anti-programmed death-ligand 1 (PD-L1) blockade in patients with non-small-cell lung cancer profiled with targeted next-generation sequencing. J Clin Oncol 36: 633-641, 2018.

53. Mazieres J, Drilon A, Lusque A, Mhanna L, Cortot AB, Mezquita L, Thai AA, Mascaux C, Couraud S, Veillon R, et al: Immune checkpoint inhibitors for patients with advanced lung cancer and oncogenic driver alterations: Results from the IMMUNOTARGET registry. Ann Oncol 30: 1321-1328, 2019.

54. Mahadevan N, Shivadasani P, Nowak J, Awad M and Sholl L: MA11. 10 Identification of mismatch repair deficient lung adenocarcinomas using targeted next-generation sequencing. J Thorac Oncol 13 (Suppl 10): S395, 2018.

55. Yuan X, Wu H, Han N, Xu H, Chu Q, Yu S, Chen Y and Wu K: Notch signaling and EMT in non-small cell lung cancer: Biological significance and therapeutic application. J Hematol Oncol 7: 87, 2014.

56. Qiu H, Zmina PM, Huang AY, Askew D and Bedogni B: Inhibiting Notch1 enhances immunotherapy efficacy in melanoma by preventing Notch1 dependent immune suppressive properties. Cancer Lett 434: 144-151, 2018.
57. Zhang K, Hong X, Song Z, Xu Y, Li C, Wang G, Zhang Y, Zhao X, Zhao Z, Zhao J, et al: Identification of deleterious mutation as novel predictor to efficacious immunotherapy in NSCLC. Clin Cancer Res 26: 3649-3661, 2020.

58. Jiang D, Niu Z, Zhang J, Wang Y, Shang L, Li B, Guo J, Wang B, Zhao LQ, Wang W, et al: Notch family gene mutations associate with high tumor mutational burden in diverse cancers. J ClinOncol 37 (Suppl 15): e14616, 2019.

59. Severson EA, Ramkissoon S, Daniel S, Vergilio JA, Gay LM, Elvin JA, Suh J, Frampton GM, Ali SM, Miller VA and Ross JS: Association of tumor mutational burden in cutaneous squamous cell carcinoma with genomic alterations in Notch family receptors. J Clin Oncol 35 (Suppl 15): e13031, 2017.

60. Subbiah V, Solit DB, Chan TA and Kurzrock R: The FDA approval of pembrolizumab for adult and pediatric patients with tumor mutational burden $(\mathrm{TMB}) \geq 10$ : A decision centered on empowering patients and their physicians. Ann Oncol 31: $1115-1118,2020$

61. Chalmers ZR, Connelly CF, Fabrizio D, Gay L, Ali SM, Ennis R, Schrock A, Campbell B, Shlien A, Chmielecki J, et al: Analysis of 100,000 human cancer genomes reveals the landscape of tumor mutational burden. Genome Med 9: 34, 2017.

This work is licensed under a Creative Commons Attribution-NonCommercial-NoDerivatives 4.0 International (CC BY-NC-ND 4.0) License. 